\title{
Changes in serum dehydroepiandrosterone, androstenedione, testosterone, and 17 $\beta$-oestradiol levels associated with disease and surgery in the horse
}

\author{
Ignacio Ayala, Nieves Martos \\ University of Murcia, Veterinary Faculty, Department of Animal Medicine \& Surgery, Murcia, Spain
}

Received March 15, 2012

Accepted October 23, 2012

\begin{abstract}
The aim of this work was to measure serum concentrations of dehydroepiandrosterone, androstenedione, testosterone and $17 \beta$-oestradiol in horses with various diseases and after surgery. We hypothesize that diseases and castration could potentially affect concentrations of steroid reproductive hormones. Blood samples were obtained from six groups of horses comprising a total of 119 horses ( 75 males and 44 females, 5-15 years old) with laminitis, acute abdominal syndrome, acute diseases, chronic diseases, after castration and healthy control. Hormone concentrations in serum were determined for each group using competitive enzyme immunoassay. Significant increases compared to control were found for dehydroepiandrosterone in horses with castration $(P<0.01)$, acute abdominal syndrome and acute diseases $(P<0.05)$. Besides, significant increases were observed for androstenedione in horses with laminitis, castration and acute diseases $(P<0.01)$, and in acute abdominal syndrome and chronic diseases $(P<0.05)$. Significant increases were also found for testosterone in horses with castration $(P<0.01)$ and with laminitis, acute abdominal syndrome and chronic diseases $(P<0.05)$. The lowest values of testosterone were found in the control group. Compared to control, 17b-oestradiol serum concentrations showed significant decreases $(P<0.01)$ in horses with laminitis, acute abdominal syndrome, acute and chronic diseases. Significant differences $(P<0.05)$ for the four studied hormones were found between males and females in each group. Our results showed that there were significant differences in steroid reproductive hormone concentrations in diseased horses and in those after surgery, compared to controls.
\end{abstract}

Equine, steroid reproductive hormone levels, serum, EIA

No detailed comparative data are available on steroid reproductive hormone concentrations in horses with various diseases and after surgery. We hypothesize that these pathological situations or surgery could potentially affect concentrations of steroid reproductive hormones. In fact, the response of the hypothalamic-pituitaryadrenal (HPA) axis and adrenal medulla has been assessed in horses with various diseases and following surgery (Ayala et al. 2011). Several HPA axis components are modulated by gonadal steroids and pregnolone is the precursor to cortisol and cortisone (Figueiredo et al. 2002).

The aim of the present study was to measure serum concentrations of steroid reproductive hormones in horses with various diseases (acute and chronic) or after surgery, whilst avoiding the potential influence of other factors (time of sampling, transport, therapy and season), and to provide data for assessing the relationship between disease and reproductive endocrine system activity.

\section{Materials and Methods}

A total of 95 Spanish-bred horses diseased or referred for castration (Table 1) admitted to the Veterinary Clinical Hospital of the University of Murcia in Spain were studied, in addition to 24 horses in the control group. Only clinical cases admitted during morning hours were selected for this study. Since the horse is a seasonal breeder, blood sample collection was performed only in spring of the same year. All mares were determined to be in the ovulatory season by ultrasonic examinations for detection of ovulation (one or more large follicles with $>30 \mathrm{~mm}$ in diameter).

Address for correspondence:

Dr. Ignacio Ayala

Department of Animal Medicine \& Surgery

Veterinary Faculty

University of Murcia, Murcia 30100, Spain
Phone: +34868 887070

Fax: +34868 884147

E-mail: iayape@um.es

http://actavet.vfu.cz/ 
Table 1. Sex and age in groups of diseased/stressed and control horses.

\begin{tabular}{lccccc}
\hline Group & $\mathrm{n}$ & Males & Females & $\begin{array}{c}\text { Mean age } \\
\text { (years) }\end{array}$ & $\begin{array}{c}\text { Age range } \\
\text { (years) }\end{array}$ \\
\hline Laminitis & 10 & 6 & 4 & 6.5 & $5-12$ \\
AAS & 43 & 31 & 12 & 7.6 & $5-15$ \\
Acute disease & 10 & 5 & 5 & 7.8 & $5-12$ \\
Chronic & & & & & \\
disease & 22 & 11 & - & 7.5 & $5-14$ \\
Castration & 10 & 10 & 32 & 7.3 & $5-10$ \\
Total & 95 & 63 & 12 & 6 & $5-15$ \\
Control & 24 & 12 & & & $5-7$ \\
\hline
\end{tabular}

AAS - acute abdomen syndrome

The animals were divided into five study groups using a diagnostic-based classification. One group suffered from acute laminitis (animals without radiographic evidence of displacement of the distal phalanx; $n=10$ ) and the second group $(n=43)$ included animals with acute abdominal syndrome (AAS), which was subdivided according to the origin of the syndrome in: colon displacement $(\mathrm{n}=18)$, proximal enteritis $(\mathrm{n}=6)$, paralytic ileus $(\mathrm{n}=$ $6)$, large intestine impactation $(n=7)$, strangulated obstruction of the small intestine $(n=4)$ and strangulated obstruction of the large intestine $(n=2)$. The third group $(n=10)$ included acute diseases (trauma, arthritis, myositis, etc.), and the fourth $(\mathrm{n}=22)$ included chronic diseases (degenerative articular disease, atlanto-occipital luxation, etc.). For this group, only cases of routine follow-up and not exacerbations were included. The fifth group $(n=10)$ included male horses that were castrated under general anaesthesia with halothane and blood sampling was performed in these horses 6-8 h after surgery. The control group $(n=24)$ included horses that were without evidence of disease both clinically and based on analysis of blood indicators.

Since hormone concentrations may be influenced by the time of sampling and other elements of the subject's environment, all blood samples were obtained under the same conditions for both control and study animals. All the horses had been transported from regional locations (for a duration of less than one hour) and were kept at the hospital for similar duration (30-60 min) before blood sampling. Also, mindful of the potential variation in concentration due to pulsatility of hormone secretion, two samples were obtained, 10 min apart. They were analysed separately and the mean value was calculated. The samples were obtained by jugular puncture $(8 \mathrm{ml})$ between 09:00 and 11:00 h, prior to the administration of any therapy, using pre-chilled vacuum tubes and extracted in less than $15 \mathrm{~s}$ to avoid changes induced by puncture stress. Serum was obtained by centrifugation in clotting tubes $\left(1500 \times \mathrm{g}, 20 \mathrm{~min}\right.$ at $\left.4{ }^{\circ} \mathrm{C}\right)$ and routine haematological and biochemical analyses were performed for each animal. The DHEA, androstenedione, testosterone and $17 \beta$-oestradiol concentrations were measured on serum samples after storing at $-70^{\circ} \mathrm{C}$ for less than two weeks. Hormones were assayed by amplified enzyme immunoassay EIA as described by Illera et al. (2003) and were expressed in nmol/1, except for $17 \beta$-oestradiol (in pmol/l).

The BMDP (Biomedical Data Program, Statistical Software Inc.) was used for statistical analysis of the results according to group and sex, using the Kolmogorov-Smirnov, pairwise $t$ and Bonferroni tests. Correlations between different indicators (haematological, biochemical and hormonal variables) were also calculated and, for all statistical comparisons, values were expressed as mean \pm standard deviation with $P \leq 0.05$ denoting significant differences.

\section{Results}

Complete blood count and biochemical analysis showed abnormal findings (hyperfibrinogenemia, neutrophilia, etc.) (Table 2) in all five groups of horses with a significant difference $(P<0.05)$ compared to the control animals. However, there were no significant differences in these indicators within the study groups (diseased animals and horses after castration), and there was no correlation with the studied hormonal concentrations, either.

Table 3 shows concentrations of the studied hormones, as well as significant differences between studied groups of horses and the control. 
Table 2. Abnormal findings of haematology in horses of different groups

\begin{tabular}{lcccc}
\hline Group & $\begin{array}{c}\text { Increased } \\
\text { fibrinogen }\end{array}$ & Leukocytosis & Leukopaenia & Neutrophilia \\
\hline Laminitis & 4 & 2 & - & 2 \\
AAS & 19 & 15 & 5 & 15 \\
Acute diseases & 5 & 2 & 4 & 2 \\
Chronic diseases & 3 & 2 & - & 1 \\
Castration & 3 & 1 & 0 & 1 \\
\hline
\end{tabular}

AAS - acute abdomen syndrome

Table 3. Mean concentrations \pm standard deviation of serum dehydroepiandrosterone, androstenedione, testosterone (nmol/l) and $17 \beta$-oestradiol (pmol/l) in horses of different groups

\begin{tabular}{llrccrc}
\hline Group & Sex* & $\mathrm{n}$ & DHEA & Androstenedione & Testosterone & $17 \beta$-oestradiol \\
\hline Control & Males & 12 & $110.2 \pm 10.9$ & $48.9 \pm 5.5$ & $6.0 \pm 1.4$ & $360.9 \pm 22.5$ \\
& Females & 12 & $47.7 \pm 7.3$ & $21.8 \pm 0.4$ & $2.4 \pm 0.3$ & $1217.7 \pm 40.8$ \\
Laminitis & Males & 6 & $256.0 \pm 24.7$ & $115.9 \pm 8.1^{\mathrm{B}}$ & $14.5 \pm 0.4^{\mathrm{A}}$ & $58.0 \pm 8.2^{\mathrm{B}}$ \\
& Females & 4 & $44.9 \pm 3.1$ & $18.9 \pm 3.3^{\mathrm{B}}$ & $5.0 \pm 0.3^{\mathrm{A}}$ & $131.6 \pm 11.4^{\mathrm{B}}$ \\
AAS & Males & 31 & $306.5 \pm 32.2^{\mathrm{A}}$ & $144.4 \pm 16.5^{\mathrm{A}}$ & $14.7 \pm 2.0^{\mathrm{A}}$ & $115.5 \pm 10.8^{\mathrm{B}}$ \\
& Females & 12 & $105.7 \pm 19.5^{\mathrm{A}}$ & $49.0 \pm 9.5^{\mathrm{A}}$ & $4.8 \pm 0.5^{\mathrm{A}}$ & $176.7 \pm 18.3^{\mathrm{B}}$ \\
Acute & Males & 5 & $292.4 \pm 26.2^{\mathrm{A}}$ & $107.3 \pm 9.5$ & $16.3 \pm 1.6$ & $56.2 \pm 6.8^{\mathrm{B}}$ \\
diseases & Females & 5 & $107.5 \pm 3.7^{\mathrm{A}}$ & $49.5 \pm 3.4$ & $3.4 \pm 0.9$ & $174.5 \pm 22.3^{\mathrm{B}}$ \\
Chronic & Males & 11 & $244.1 \pm 18.1$ & $108.2 \pm 17.9^{\mathrm{A}}$ & $13.1 \pm 1.7^{\mathrm{A}}$ & $16.6 \pm 34.0^{\mathrm{B}}$ \\
diseases & Females & 11 & $79.1 \pm 17.5$ & $44.5 \pm 9.0^{\mathrm{A}}$ & $4.3 \pm 0.7^{\mathrm{A}}$ & $312.0 \pm 29.4^{\mathrm{B}}$ \\
Castration & Males & 10 & $228.3 \pm 13.0^{\mathrm{B}}$ & $102.7 \pm 7.5^{\mathrm{B}}$ & $8.4 \pm 0.3^{\mathrm{B}}$ & $411.8 \pm 27.3$ \\
\hline
\end{tabular}

AAS - acute abdomen syndrome, ${ }^{\mathrm{A}} P<0.05,{ }^{\mathrm{B}} P<0.01$ - significant differences with respect to the control group, * significant differences $(P<0.05)$ for the four studied hormones were found between males and females in each group

DHEA - Dehydroepiandrosterone

Significant differences were found for testosterone and oestradiol for the different types of AAS. The highest mean concentration of testosterone for the AAS group was found in horses with strangulated obstruction of the small intestine and the lowest values in animals with colon impactation. On the contrary, the highest mean concentration of oestradiol was observed in proximal enteritis and the lowest in strangulated obstruction of the small intestine.

Significant differences $(P<0.05)$ were found between males and females of each group for the four studied hormones (DHEA, androstenodione, testosterone and 17b-oestradiol) but there was no association with age.

\section{Discussion}

The finding of abnormal haematological and biochemical indicators in all the study horses is consistent with the underlying inflammatory component of the diseases with which they were afflicted and many of these indicators, including hyperfibrinogenaemia and neutrophilia are frequently measured as nonspecific indicators of inflammation (Auer et al. 1989). Unsurprisingly, therefore, there were no significant differences in these indicators between the study groups.

Significant changes in serum concentrations of DHEA and androstenedione were observed 
in several disease groups compared to controls. The DHEA was shown to be triggered by stress-induced release of ACTH and also to exhibit memory-enhancing, antidepressant, anxiolytic and antiaggression properties (Compagnone and Mellon 2000). Although several authors have shown that major stress reduces DHEA concentrations (Fenster et al. 1997), our results are similar to those that have provided empirical evidence that the DHEA concentration is increased by acute stress in healthy humans and that the DHEAcortisol ratio may index the degree to which an individual is buffered against the negative effects of stress (Morgan et al. 2004).

Only moderate and non-significant differences were found in horses with chronic diseases, consistent with the elevated cortisol concentration and relatively decreased circulating and urinary excretion of DHEA in a variety of severe chronic illnesses in humans (Parker et al. 1985). The DHEA is involved in adaptative reactions and improves the body's resistance to stress-induced pathologies (Obut et al. 2003). A decrease of androgen serum concentrations may lead to a more proinflammatory disease state because DHEA has been shown to inhibit proinflammatory cytokines such as secretion of tumour necrosis factor (Straub et al. 2002). Significant differences were found between males and females for both DHEA and androstenodione both in the diseased and control groups. Human males have higher circulating concentrations of DHEA than females, with higher maximally stimulated DHEA concentrations that can be achieved after short term ACTH stimulation (Parker 1999) although the mechanism responsible for this accentuated production of DHEA is unclear.

Significant increase in testosterone was observed between groups with laminitis, AAS and chronic disease, compared to control. Similarly, increases in testosterone concentrations were found in response to a nociceptive stimulus in both sexes in humans (Craft et al. 2004). However, other authors have shown different results, demonstrating decreased serum testosterone to be one of the first signs of stress in the man (Fenster et al. 1997). Thus, although there are several lines of preclinical and clinical data suggesting that circulating epinephrine may influence plasma testosterone concentrations, the direction of this response is inconsistent (Elman et al. 2001) which may be due to the nature of stress. Highly significant increase of testosterone was found between the group of horses after castration and control group which could be related to a marked increase in adrenal androgen secretion induced by surgical stress.

In contrast to other studied variables, the highest concentrations of $17 \mathrm{~b}$-oestradiol were found in the control group. Catecholamines increase the number of oestradiol receptors in the hypothalamus which has implications for the interaction between stress and GnRH-LH suppression (Dobson et al. 2003). Interruption of LH pulsatility (by GnRH antagonists or by stressors) in the follicular phase lowers oestradiol production and reduces the subsequent rate of blastocyst formation (Dobson and Smith 1998). Delayed LH surges and resulting extended follicular phases have an adverse effect on fertility (Dobson and Smith 1998). Perhaps a similar mechanism may be associated with disease conditions and surgery in the horse, although this fact was not evaluated in the present work.

We observed decreased 17b-oestradiol serum concentrations in the group of horses with chronic disease. In a similar way, decreased oestradiol concentrations in rats were found under different types of chronic stress (Andersen et al. 2004). The ACTH secretion is subjected to negative feedback control by glucocorticoids, and $17 \mathrm{~b}$-oestradiol has been strongly implicated in reduced efficacy of glucocorticoid negative feedback inhibition (Burgess and Handa 1992) and this could result in a greater $\mathrm{ACTH}$ response and subsequent corticosterone secretion. No significant differences were found between horses after castration and the control group. The higher concentrations of oestradiol found in the former group could be due to several factors that may potentially decrease the HPA response and therefore influence the 
hypothalamic-pituitary-gonadal axis, such as the short duration of surgery and low degree of surgical manipulation (Traynor and Hall 1981), as well as the use of analgesics before surgery (Sanhouri et al. 1992).

Significant changes were observed for testosterone and oestradiol for different types of AAS, reflecting that strangulating colics affect the steroid reproductive hormone profile (higher testosterone and lower oestradiol concentrations) in a different way to non strangulating colics (lower testosterone and higher oestradiol concentrations). The AAS in the horse is not a homogeneous condition and it may be associated with varying degrees of gastrointestinal function disturbances including hypovolaemic shock, dehydration, acid-base and electrolyte abnormalities and endotoxaemia (Santschi et al. 1991).

We have documented significant increases in DHEA, androstenedione, and testosterone, and significant decreases in $17 \beta$-oestradiol concentrations compared to control animals in diseased horses and in those after castration. These findings may improve our knowledge of the relationship between disease/surgery and reproductive endocrine system activity in the horse.

\section{References}

Andersen ML, Bignotto M, Machado RB, Tufik S 2004: Different stress modalities result in distinct steroid hormone responses by male rats. Braz J Med Biol Res 37: 791-797

Auer DE, Ng JC, Thompson HL, Inglis S, Seawright AA 1989: Acute phase response in horses: Changes in plasma cation concentrations after localised tissue injury. Vet Record 124: 235-239

Ayala I, Martos NF, Silvan G, Gutierrez-Panizo C, Clavel JG, Illera JC 2011: Cortisol, adrenocorticotropic hormone, serotonin, adrenaline and noradrenaline serum concentrations in relation to disease and stress in the horse. Res Vet Sci 2; E-pub ahead of of print, PMID: 21641009.

Burgess LH, Handa RJ 1992: Chronic estrogen-induced alterations in adrenocorticotropin and corticosterone secretion, and glucocorticoid receptor-mediated functions in female rats. Endocrinology 131: 1261-1269

Compagnone NA, Mellon SH 2000: Neurosteroids: biosynthesis and function of these novel neuromodulators. Front Neuroendocrinol 21: 1-56

Craft R, Mogil JS, Aloisi AM 2004: Sex differences in pain and analgesia: the role of gonadal hormones. Eur J Pain 8: 397-411

Dobson H, Ghuman S, Prabhakar S, Smith R 2003: A conceptual model of the influence of stress on female reproduction. Reproduction 125: 151-163

Dobson H, Smith RF 1998: Stress and subfertility. Reprod Dom Anim 33: 107-111

Edquist LE, Forsberg M 2008: Clinical reproductive endocrinology. In: Kaneko JJ, Harvey JW, Bruss ML (Eds): Clinical Biochemistry of Domestic Animals. $6^{\text {th }}$ edn. Academic Press, London, pp. 589-617

Elman I, Goldstein DS, Adler CM, Shoaf SE, Breier A 2001: Inverse relationship between plasma epinephrine and testosterone levels during acute glucoprivation in healthy men. Life Sciences 68: 1889-1898

Fenster L, Katz DF, Wyrobek AJ, Pieper C, Rempel DM, Oman D, Swan SH 1997: Effects of psychological stress on human semen quality. J Androl 18: 194-202

Figueiredo HF, Dolgas CM, Herman JP 2002: Stress activation of cortex and hippocampus is modulated by sex and stage of estrus. Endocrinology 143: 2534-2540

Illera JC, Silvan G, Munro CJ, Lorenzo PL, Illera MJ, Liu IK, Illera M 2003: Amplified androstenedione enzymeimmunoassay for the diagnosis of cryptorchidism in the male horse: comparison with testosterone and estrone sulphate methods. J Steroid Biochem Mol Biol 84: 377-382

Morgan CA 3rd, Southwick S, Hazlett G, Rasmusson A, Hoyt G, Zimolo Z, Charney D 2004: Relationships among plasma dehydroepiandrosterone sulfate and cortisol levels, symptoms of dissociation, and objective performance in humans exposed to acute stress. Arch Gen Psychiatry 61: 819-825

Obut TA, Ovsyukova MV, Cherkasova OP 2003: Stress-limiting effect of dehydroepiandrosterone sulfate and its mechanism. Bull Exp Biol Med 3: 231-233

Parker Jr, R 1999: Dehydroepiandrosterone and dehydroepiandrosterone sulfate production in the human adrenal during development and aging. Steroids 64: 640-647

Parker L, Levin E, Lifrak E 1985: Evidence for adrenocortical adaptation to severe illness. J Clin Endocrinol Metab 60: 947-952

Sanhouri AA, Jones RS, Dobson H 1992 : Effects of xylazine on the stress response to transport in male goats. Brit Vet J 148: 119-127

Santchi EM, Leblanc MM, Weston PG 1991: Progestagen, oestrone sulphate and cortisol concentrations in pregnant mares during medical and surgical disease. J Reprod Fertil Suppl 44: 627-634 
Straub RH, Lehle K, Herfart H 2002: Dehydroepiandrosterone in relation to other adrenal hormones during an acute inflammatory stressful disease compared with chronic inflammatory disease: role of interleukin-6 and tumour necrosis factor. Eur J Endocrinol 146: 365-374

Traynor C, Hall GM 1981: Endocrine and metabolic changes during surgery: anaesthetic implications. Brit J Anaesth 53: 153-160 